\title{
DIFFERENCES IN ACTIVATION OF THE VASTUS LATERALIS MUSCLE DURING MAXIMAL EXERCISE TEST BETWEEN VERY-LOW-BIRTH- WEIGHT PRETERM AND FULL-TERM CHILDREN
}

original paper

(c) University School of Physical Education in Wroclaw

DOI: https://doi.org/10.5114/hm.2020.91343

\section{JOSY DAVIDSON ${ }^{1}$, SABRINA PINHEIRO TSOPANOGLOU ${ }^{1}$, VICTOR ZUNIGA DOURADO ${ }^{2}$, NELSON CARVAS JR ${ }^{3}$, AMÉLIA MIYASHIRO NUNES DOS SANTOS ${ }^{1}$, ANA LUCIA GOULART ${ }^{1}$, DIRCEU SOLÉ ${ }^{1}$}

${ }^{1}$ Department of Pediatrics, Federal University of São Paulo, São Paulo, Brazil

${ }^{2}$ Department of Human Movement Sciences, Laboratory of Human Movement, Federal University of São Paulo, Santos, Brazil

${ }^{3}$ Department of Post Graduation, IAMSPE, São Paulo, Brazil

\section{ABSTRACT}

Purpose. To compare the pattern of the electrical activation of the vastus lateralis muscle during a maximal exercise test between children born prematurely and full-term.

Methods. The study was conducted in preterm children born at less than 37 weeks of gestational age with a birth weight of less than $1500 \mathrm{~g}$ aged 6-9 years compared with children born at term of the same age. A rapidly incremental exercise test on a treadmill was performed, and surface electromyography signals of the vastus lateralis muscle were collected throughout the test. The root mean square (RMS) was calculated every minute. The Mann-Whitney and Friedman's tests were applied to verify the differences in each minute between and within groups, and nonlinear regression analysis was performed to establish the factors associated with the pattern of vastus lateralis activation.

Results. Overall, 68 children were analysed: 37 in the premature group and 31 in the term group. The preterm group showed higher RMS values than the full-term group throughout the test, with significant differences at rest and in the 3rd, 4 th, 5th, and 6th minutes of the exercise test. Particularly, the preterm group presented $28.5 \%$ higher RMS values than the term group throughout the analysed period.

Conclusions. The lower limbs of the children born prematurely showed higher excitability than those of the children born at full-term. These data could help maintain and/or improve their physical ability during exercise tests.

Key words: surface electromyography, premature, infant, exercise tests, term birth, child

\section{Introduction}

Within the previous 3 decades, the number of preterm births has increased worldwide. Advances in perinatal assistance have contributed to a gradual increase in the survival rates of affected infants [1,2]. Despite that, prematurity has been considered an important cause of neonatal morbidity due to neurological and respiratory complications that can lead to clinical repercussions during childhood, adolescence, and even adulthood. Studies have shown that children and adolescents born prematurely, especially those who had bronchopulmonary dysplasia, present decreased exercise capacity during exercise compared with those born prematurely without respiratory diseases or those born at term [3].

Recently, a study on children followed up in our service has reported that very-low-birth-weight preterm children walked a shorter distance in a 6-min walk test than those born at term [4]; this result might be attributable to such factors as pulmonary and cardiovascular conditions.

Despite the importance of pulmonary and cardiovascular conditions for exercise capacity, peripheral

Correspondence address: Josy Davidson, Department of Pediatrics, Neonatal Division, Federal University of São Paulo, Rua Marselhesa, 630 ZIP code 04020-060 - Vila Clementino, São Paulo, SP, Brazil, e-mail: josydavidson@yahoo.com.br

Received: April 29, 2019

Accepted for publication: October 11, 2019

Citation: Davidson J, Tsopanoglou SP, Dourado VZ, Carvas Jr N, dos Santos AMN, Goulart AL, Solé D. Differences in activation of the vastus lateralis muscle during maximal exercise test between very-low-birth-weight preterm and full-term children. Hum Mov. 2020;21(3):31-38; doi: https://doi.org/10.5114/hm.2020.91343. 
muscles can also play an important role in exercise limitation [5]. Surface electromyography is a non-invasive and reliable method for assessing muscle activity, which enables the evaluation of muscle recruitment during exercise [6, 7].

It has been suggested that decreased exercise capacity is linked to a lower lung capacity in children born prematurely. However, studies that evaluated the influence of peripheral muscles on exercise capacity are scarce or non-existent. Therefore, we aimed to determine the electrical activity of the lower limbs in preterm children and compare their outcomes with those of children born at term.

\section{Material and methods}

\section{Participants and study design}

We conducted a cross-sectional study among preterm children born less than 37 weeks of gestational age with a birth weight of less than $1500 \mathrm{~g}$ aged 6-9 years who were regularly followed up in a paediatric outpatient clinic. These preterm children were compared with a group of homogeneous children born at term (most of them were relatives or friends of the preterm children followed in our service).

The inclusion criteria were as follows: any sex and stable respiratory conditions, i.e., no wheezing in the previous 2 weeks and/or no acute lung disease diagnosed by physicians.

Children with neurological and/or gait impairment in physical examination findings and/or cognitive impairment that might prevent the understanding and/ or the execution of the test, those with congenital malformations and neuromuscular diseases, those who had been hospitalized in the previous 3 months, those with tachycardia and/or hypertension [8] at rest, and those that did not show up to the appointment after 4 attempts to perform the test were excluded.

The assessments were performed in a single session, previously scheduled with the parents or guardians. Data on birth, physical activity (ordinary school classes and extra classes, on-foot transportation, and games that required movement), and health conditions until the day of the test were recorded on the basis of the hospital chart and parent interview findings.

\section{Exercise test}

The children were instructed to wear comfortable clothes and shoes on the day of the test. They were also advised to avoid any vigorous physical activity for at least $2 \mathrm{~h}$ before the test. All tests were conducted in the morning by 2 previously trained researchers and were supervised by a physician.

Prior to the test, we measured the weight and height of the children using an eye-level mechanical beam physician scale with a height rod (Filizola PL200, São Paulo, Brazil). Next, the children remained seated on a chair for 10 min before starting the test. Thereafter, we measured their blood pressure, heart and respiratory rates, and degree of dyspnoea at rest using a visual analogue scale [9].

We considered the exercise test as maximum when at least $85 \%$ of the predicted maximum heart rate (HRmax) was reached, calculated as:

$$
H R \max =210-\left(0.65 \times \text { age }_{\mathrm{y}}\right)[10] .
$$

We performed an adapted exercise test in the present study. A lower and mobile handrail was fixed on the treadmill, allowing maintenance of a shoulder flexion of $30-45^{\circ}$; the participants' hands were supported throughout the test.

During warm-up, the walking velocity was set at $1 \mathrm{mph}$ during the first $3 \mathrm{~min}$. In the $4^{\text {th }}$ minute, the velocity of the treadmill was progressively increased up to $3.5 \mathrm{mph}$ for children aged 6-7 years and $4.0 \mathrm{mph}$ for those aged 8-9 years, without any inclination during the $1^{\text {st }}$ minute of the exercise. Then, we increased the inclination by $2 \%$ every minute until the grade of $10 \%$ was reached $[5,11]$. If a child had not reached the maximum effort, we kept the inclination constant and increased the speed by $0.5 \mathrm{mph}$ every minute until exhaustion.

The test was interrupted in the presence of intense fatigue (EVA scale score of $>7$ ) [9] when the participant requested to stop the test or upon reaching $85 \%$ of the predicted HRmax.

\section{Preparation, collection, and processing of electromyography signals}

Electromyography signals were collected and processed by using the electromyography 830C EMG system $^{\circledast}$ (São José dos Campos, Brazil). The frequency for the acquisition of the electromyography recordings was $2 \mathrm{kHz}$ with an amplifier gain of 2000 . Artefacts and noise were treated with a band pass filter of $4 \mathrm{~Hz}$ and cut-off frequencies of $20-500 \mathrm{~Hz}$, impedance system of $10^{9} \mathrm{ohms}$, noise ratio $<3 \mu \mathrm{V} \mathrm{RMS}$, and a common rejection mode ratio of less than $120 \mathrm{~dB}$. Beyond these filters, the computer was maintained switched off during the entire data acquisition, and 
the proximal wires of signal acquisition were fixed with an elastic band around the thigh to reduce movement artefacts. All data were acquired and processed by using a 16-bit analogue-to-digital converter. The skin was lightly shaved and cleaned with $70^{\circ} \mathrm{GL}$ alcohol wipes, followed by slight abrasion to reduce skin impedance. A self-adhesive silver/silver chloride with conductive hydrogel composition surface and 4.2-mm diameter Medi-Trace ${ }^{\circledast} 100$ (Kendall; Mansfield, Canada) were used to connect the equipment to the child's thigh and placed in a bipolar configuration over the vastus lateralis (VL) muscle in accordance with the Surface Electromyography for the Non-Invasive Assessment of Muscles [12]. A single researcher conducted all the electrode assessments and muscle palpation, and a series of isometric contractions for the specific muscle were also used for signal validation.

Data were collected during rest, warm-up, and exercise and compiled with a mathematical routine developed for the study, in which the root mean square (RMS) was calculated by using the mean of every first $30 \mathrm{~s}$ of each minute of the exercise test. RMS of the warm-up period was calculated on the basis of its last $30 \mathrm{~s}$. For comparison of the measurements among all participants, the exercise stage was evaluated until the $6^{\text {th }}$ minute; in those who continued the test, the last minute of the exercise stage (final) was also analysed.

\section{Statistical analysis}

The descriptive data of the sample were presented as means and standard deviations or as medians (interquartile ranges). For categorical variables, we calculated the frequencies and percentages. Comparisons between groups were performed by using Student's $t$-test or Mann-Whitney test for continuous variables, depending on the symmetrical or asymmetrical distribution, and by using the chi-squared test or Fisher's exact test for categorical variables.

The normality of the continuous data was evaluated with the Kolmogorov-Smirnov test. As for the electromyography signals presenting asymmetrical distribution, the Mann-Whitney test was applied, followed by 10,000-case Monte Carlo simulation to verify the differences between groups in each minute of the exercise test. Repeated measures were contrasted by using the Friedman test, and differences between the measures in each group were tested with the Wilcoxon test; $p$ values were adjusted in accordance with the number of repetitions of the measures.

To identify significant predictors at the level of the logarithm of the measurements (level 1) and children (level 2), a nonlinear regression analysis was performed to determine the best model by using the R Project software, lme4 package, version 3.2.3. The step-up strategy was applied in accordance with the simpler model (components of variance) to a more complex model (inter-level interaction). The significance of the quality model was evaluated in accordance with the difference in the log-likelihood ratio. Intra-class correlation coefficients (ICC) of random effects [intercept / (intercept + residual)] and fixed effects [intercept / (intercept + time)] were estimated to determine the variability of muscle activity among the stages of the exercise test, children and preterm groups, and the interaction between them. The significance of the fixed measures was established in the lmerTest package. The probability of an alpha error was set at $5 \%$.

\section{Ethical approval}

The research related to human use has complied with all the relevant national regulations and institutional policies, has followed the tenets of the Declaration of Helsinki, and has been approved by the Research Ethics Committee of the Federal University of São Paulo.

\section{Informed consent}

Informed consent has been obtained from all individuals included in this study, as well as from their legal guardians.

\section{Results}

A total of 93 children were recruited in the study. However, 9 (9.6\%) children in the preterm group did not accept study participation or withdrew after 4 attempts. Among the 84 remaining children, 13 (15.8\%) were excluded owing to operational problems during the surface electromyography evaluation (4 in the term group and 9 in the preterm group); 2 (2.3\%) prematurely interrupted the exercise test, which suggested poor effort; and $1(1.2 \%)$ child in the term group presented tachycardia at rest. Finally, 68 (73.9\%) children were analysed: 37 in the premature group and 31 in the term group.

There were no differences in the general conditions, level of physical activities, or complications after neonatal discharge between the groups at the time of the study (Table 1).

We observed lower RMS values in the preterm group throughout the test (Figure 1). The within-group analysis during the exercise test showed that both groups had increased RMS values in all stages compared with those at rest or during warm-up. 


\section{HUMAN MOVEMENT}

J. Davidson et al., Peripheral muscle pattern in preterm children

Table 1. General conditions of the sample

\begin{tabular}{lccc}
\hline Characteristics & $\begin{array}{c}\text { Premature group } \\
(n=37)\end{array}$ & $\begin{array}{c}\text { Term group } \\
(n=31)\end{array}$ & $p$ value \\
\hline Gestational age (weeks) & $30.23 \pm 1.20$ & $39.09 \pm 2.72$ & $<0.001$ \\
Age at study day (years) & $7.70 \pm 1.04$ & $7.87 \pm 1.18$ & 0.541 \\
Current weight (kg) & $26.11 \pm 6.25$ & $28.6 \pm 7.56$ & 0.139 \\
Current height (m) & $1.25 \pm 0.87$ & $1.28 \pm 0.95$ & 0.150 \\
Current BMI $\left(\mathrm{kg} \cdot \mathrm{m}^{-2}\right)$ & $16.71 \pm 2.51$ & $16.74 \pm 2.64$ & 0.953 \\
Female, $n$ (\%) & $14(37.8)$ & $16(51.7)$ & 0.255 \\
Physical activity/week (min) & $198 \pm 84$ & $228 \pm 94$ & 0.190 \\
No physical activity & $17(46.0)$ & $13(35.1)$ & 0.383 \\
\hline
\end{tabular}

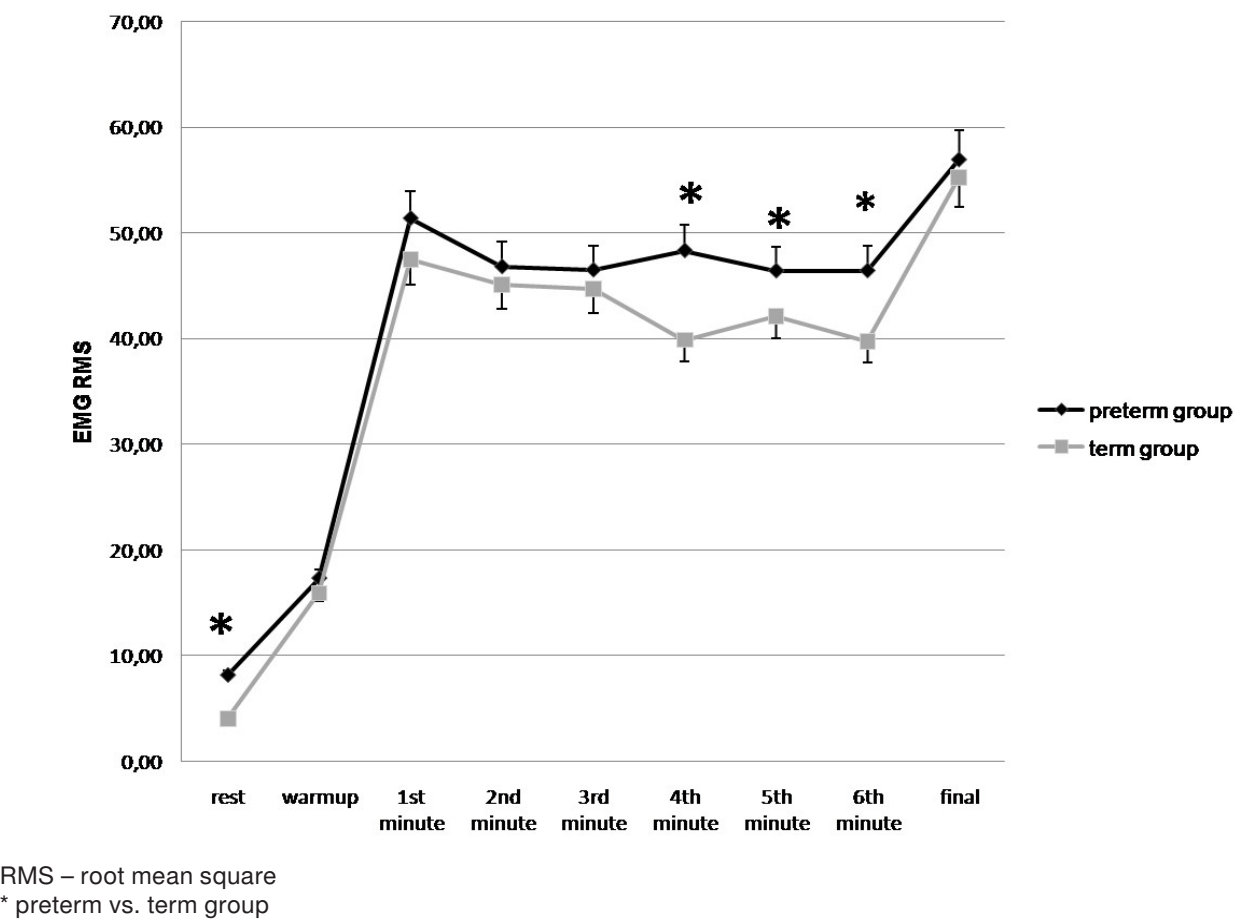

Figure 1. Between-group analysis during the exercise test in all stages

Table 2. Within-group analysis of surface electromyography signals of vastus lateralis

\begin{tabular}{lcc}
\hline RMS & $\begin{array}{c}\text { Preterm group } \\
(n=37)\end{array}$ & $\begin{array}{c}\text { Term group } \\
(n=31)\end{array}$ \\
\hline Rest & $8.15(6.95 ; 12.38)^{*}$ & $\begin{array}{c}4.01(4.33 ; 7.39)^{*} \\
15.92(-21.55 ; 14.24)^{*}\end{array}$ \\
Warm-up & $17.31(18.80 ; 33.88)^{*}$ & $47.46(43.82 ; 91.14)^{+\&}$ \\
$1^{\text {st }}$ minute & $51.35(53.76 ; 97.40)^{\&}$ & $45.08(40.47 ; 60.55)$ \\
$2^{\text {nd }}$ minute & $46.78(52.27 ; 113.87)$ & $47.88(-61.14 ; 40.88)^{\$}$ \\
$3^{\text {rd }}$ minute & $46.47(-117.28 ; 51.60)$ & $44.98(38.45 ; 55.29)^{\$}$ \\
$4^{\text {th }}$ minute & $48.30(51.58 ; 116.93)$ & $44.27(37.79 ; 55.32)^{\#}$ \\
$5^{\text {th }}$ minute & $46.39(50.93 ; 116.20)^{\$}$ & $39.71(-52.63 ; 37.02)^{\# ¥ \$}$ \\
$6^{\text {th }}$ minute & $46.40(-115.40 ; 50.21)^{\# \$}$ & $55.23(50.82 ; 81.90)^{\# ¥+}$ \\
Final & $58.89(61.64 ; 114.63)^{\#+}$ & \\
\hline
\end{tabular}

RMS - root mean square

${ }^{*} \neq$ rest and warm-up vs. exercise stage, ${ }^{*} \neq 1^{\text {st }}$ minute, ${ }^{¥} \neq 3^{\text {rd }}$ minute, ${ }^{+} \neq 5^{\text {th }}$ minute, ${ }^{\&} \neq 6^{\text {th }}$ minute, ${ }^{\$} \neq$ final 
Table 3. Nonlinear regression analysis of the RMS pattern of vastus lateralis

\begin{tabular}{|c|c|c|c|c|}
\hline & Model 1 & Model 2 & Model 3 & Model 4 \\
\hline \multicolumn{5}{|l|}{ Fixed effects } \\
\hline Intercept & $3.074(0.085)^{* *}$ & $2.919(0.105)^{* *}$ & $2.895(0.123)^{* *}$ & $2.895(0.118)^{* *}$ \\
\hline Time & $0.082(0.011)^{* *}$ & $0.082(0.011)^{* *}$ & $0.087(0.011)^{* *}$ & $0.087(0.016)^{* *}$ \\
\hline Groups & & $0.285(0.118)^{*}$ & $0.329(0.167)$ & $0.329(0.160)^{*}$ \\
\hline Time * Groups & & & $-0.008(0.021)^{\mathrm{NS}}$ & $-0.008(0.022)^{\mathrm{NS}}$ \\
\hline \multicolumn{5}{|l|}{ Random effects } \\
\hline Intercept & $0.189(0.434)$ & $0.172(0.414)$ & $0.172(0.414)$ & $0.130(0.360)$ \\
\hline Residual & $0.644(0.803)$ & $0.644(0.803)$ & $0.645(0.803)$ & $0.644(0.802)$ \\
\hline Slope & & & & $0.00009714(0.009)$ \\
\hline \multicolumn{5}{|l|}{ Setting quality } \\
\hline AIC & 1730.0 & 1726.3 & 1728.2 & 1731.4 \\
\hline $\mathrm{BIC}$ & 1748.1 & 1748.9 & 1755.3 & 1767.6 \\
\hline Deviance & 1722.0 & 1716.3 & 1716.2 & 1715.4 \\
\hline
\end{tabular}

Variance (standard deviation). Values adjusted for preterm birth, weight, height, sex, and physical activity.

RMS - root mean square, AIC - Akaike information criterion, BIC - Bayesian information criterion, NS - not significant ${ }^{*} p<0.05, * * p<0.001$

However, during the subsequent increase in grade, the preterm group presented a linear pattern, while the term group showed a significant variation in electrical activity, with a shallower slope in the $6^{\text {th }}$ minute (maximum grade of the treadmill). At the end of the test, we observed the highest velocity and RMS values (Table 2).

In the multivariate analysis adjusted by prematurity, weight, height, sex, and physical activity, the best model involved the interaction between the electrical activity pattern and groups. In model 1 , we observed an RMS value of $22.65 \%$ (ICC $=0.2265$ ) among the children. During the test, we noted an increase of $8.2 \%$ $(\mathrm{ICC}=0.0820)$ in the RMS value per minute among all children.

The interaction between the electrical activity pattern and groups throughout the test showed that the preterm group had 28.5\% higher RMS values than the term group (model 2). In models 3 and 4, the analysis among the children (model 3) and groups (model 4) presented no interaction among the factors (Table 3).

\section{Discussion}

To our knowledge, this study is one of the first ones to evaluate the electrical activity of the lower limbs in children born prematurely without neurological impairment. We found that prematurity played an important role in neuromuscular conditions, even in healthy children and long-term observation, regardless of the main confounders.
In the present study, we enrolled children with a higher risk of morbidity and mortality in both the neonatal and childhood periods (i.e., most of them were born less than 34 weeks of gestational age). Therefore, our participants could show most striking characteristics of prematurity.

We observed that the children born prematurely presented a higher electrical activity of the VL muscle at rest; the activity remained higher than that in children born at term throughout the exercise, especially when the increments of grade were imposed. The higher RMS values in the preterm group suggest a greater recruitment of the muscle fibres of the VL muscle even at baseline conditions. Studies that evaluated the body position of preterm infants during neonatal intensive care unit stay have shown that the inherent hypotonia in prematurity associated with an inappropriate posture in the incubator leads to a flattened posture with predominance of extension, extreme abduction, and external rotation of the hip, demonstrating an imbalance between the abductor and adductor muscles of the hip [13, 14]. It is known that the abductor muscles of the thigh are more powerful and have more phasic actions, while the femoral rectus muscle is more tonic and more responsible for postural maintenance control $[15,16]$. It is possible that the lesser tone during the neonatal period required a greater recruitment of the muscle fibres of the abductor muscles for the acquisition of the curl posture, which could have resulted in a greater electrical excitation threshold during motor development. 
J. Davidson et al., Peripheral muscle pattern in preterm children

Another explanation for the higher electrical activity threshold in preterm infants could be the imbalance between the thigh muscles associated with the excessive flexion of the hip in the prone position that causes shortening of the iliopsoas muscle [14], responsible for the internal rotation of the hips [17]. Physiologically, valgus knees are common in children aged 3-7 years, since they help maintain body balance in the orthostatic posture. Preterm infants and children begin their motor development with lesser tone musculature and present an imbalance between the abductor and adductor muscles associated with the shortening of the iliopsoas muscle, which often causes valgus knees with knee hyperextension and hip internal rotation [17]. Thereby, there would be a greater stretching of the VL muscle, generating greater force contraction during activity (length-tension relationship). Empirically, through visual inspection (not described in the results), we observed a higher frequency of this pattern in the preterm group, which worsened during the increment of load, reinforcing the theory of antagonist muscle imbalance.

Some differences in the development of muscle activity between the groups must be pointed out. As expected, we observed that the electrical activity significantly increased in the warm-up period (walk, $1 \mathrm{mph}$ ) and in the $1^{\text {st }}$ minute of exercise when compared with that at rest in both groups. In the following periods, i.e., during grade increase, the muscle activity of the preterm and term groups became distinct. While the RMS values of the preterm group remained stable, the values in the term group significantly varied until grade $10^{\circ}$. Physiologically, it is expected that the increased RMS values until exhaustion and/or muscle fatigue would eventually decrease [18, 19]. However, this performance was not observed in both groups, mainly in the preterm group. Some factors might contribute to these findings. In high-performance individuals, including athletes, studies have demonstrated that the threshold of muscle fibre excitation increases as a result of training; this would keep muscle cell excitability levels constant even when the load increases [20]. This could occur in preterm infants for two reasons. Firstly, because of the early muscle effort to maintain the correct posture in the supine position during the neonatal phase. The constant recruitment of motor units might have increased the ability to maintain the recruitment of these motor units [19] and, consequently, the electrical excitability threshold. The second explanation we could infer is that the level of motor stimulation in preterm infants applied in outpatient follow-up settings and at home by parents could improve their neurodevelopmental conditions, allowing an excitation mechanism similar to that occurring in the muscles of training athletes.

Data were collected in the nonlinear model analysis to determine the influence of confounding factors on the electromyography pattern. The model adjusted for weight, age, height, and physical activity (data not shown) indicated that prematurity was the most important factor of increased electrical activity in the lower limbs, which corroborates the data presented earlier.

The option for a nonlinear model analysis was selected on the basis of the data variability among the children. It was observed that the electrical signal variability rate among the children was $22.65 \%$, a precondition for this kind of analysis model (values greater than 10\%) [21]. As previously mentioned, factors such as sex, age, weight, height, and physical activity were adjusted for; however, they were not shown to be primary predictors. As such, only the interaction model between the electromyography data and preterm and term groups was included. In model 2, when the effect of the groups during the exercise was included, it was observed that both stage and group effects were statistically significant. However, we noted that the electrical values (RMS) in the preterm group were $28.5 \%$ higher than those in the term group, strengthening the data obtained from the univariate analysis (Figure 1).

The options for the VL analysis and the incremental treadmill test should be discussed; the selection was based on two factors. The first one was related to the type of muscle action. The quadriceps muscles are the main muscles responsible for gait; therefore, they are the most recruited muscles in the lower limbs. Specifically, in relation to the option for the VL muscle, as a monoarticular and phasic muscle, its main function is to generate strength [16]. Thus, it is the most appropriate muscle to assess muscle recruitment during incremental tests requiring increased effort. The second factor was technical issues. One of the greatest problems in the accomplishment of this examination was the difficulty in isolating a single muscle. The use of two electrodes requires a relatively large contact area. In the case of small muscles, there is a risk of capturing crossed signals between the muscles, i.e., crosstalk [6]. In children, the isolation of a single muscle becomes even more complex owing to their size. Considering that VL is the largest quadriceps muscle, the risk of crosstalk was minimized.

The option for the incremental treadmill test was selected on the basis of the need to achieve the maximum effort in the shortest possible time. The best test 
to check the physical capacity with a focus on respiratory capacity is Balke's test, where the physician increases the slope, while maintaining the speed of the treadmill. However, during the pilot study, we observed that increments above grade $10^{\circ}$ caused lower limb discomfort due to constant knee flexion during gait, which led to earlier interruption of the test without the maximum effort being reached.

We were very concerned about the influence of the growth and developmental status, mainly due to sexual maturation and its impact on bone and muscle mass growth. Thus, we decided to include only children aged 6-9 years so the group did not involve participants in advanced sexual maturation.

\section{Study limitations}

The convenience sample selection might have influenced the results. The fact that we included children followed up in our clinic allowed us to infer that these children underwent long-term follow-up by health professionals; further, their parents might be more attentive to any significant change in their clinical conditions. It is then possible that we evaluated preterm children with above-average conditions. Conversely, the fact that we selected the brothers and friends of the children born prematurely as controls might minimize this bias, since both groups undertook similar activities in the majority of time.

Another important factor that should be considered is the level of physical activity performed by the children. Physical activity questionnaires are widely applied in epidemiological studies owing to their ease of use and low cost; however, they do not constitute the best method to assess physical activity levels [22]. In an attempt to reduce this bias, we calculated the time of physical activity inside and outside the school, in addition to the time of the children's walk to school. We could then have an idea of the general terms and conditions of their daily life activity mainly because more than $1 / 3$ of the children (preterm and term group) did not do any physical activity.

\section{Practical application of the results}

Prematurity remains a challenge for parents and health professionals, and its long-term consequences are still being studied, especially after the introduction of antenatal methods and most suitable and kinder treatments during hospitalization in the neonatal period.

The results of this study showed that although preterm children had no important motor developmen- tal impairment, they presented a different electrical pattern of lower limbs, which should be better understood. On the other hand, we reported that these children could reach a condition equivalent to that in children born at term through greater muscle stimulation.

\section{Conclusions}

In conclusion, the lower limbs in children born prematurely showed higher excitability than in children born at full-term, as well as presented a different pattern of excitability and the response of thigh muscle. These data could help maintain and/or improve the physical ability during exercise tests in this group.

\section{Acknowledgements}

The authors express special thanks to the Coordination for the Improvement of Higher Education Personnel (CAPES, Coordenação de Aperfeiçoamento de Pessoal de Nível Superior), Brazil, for the support for this project.

\section{Disclosure statement}

Josy Davidson was supported by CAPES as a postgraduate student (number 007423/2011-90). No author has any financial interest or received any financial benefit from this research.

\section{Conflict of interest}

The authors state no conflict of interest.

\section{References}

1. Grisaru-Granovsky S, Reichman B, Lerner-Geva L, Boyko V, Hammerman C, Samueloff A, et al. Populationbased trends in mortality and neonatal morbidities among singleton, very preterm, very low birth weight infants over 16 years. Early Hum Dev. 2014;90(12):821827; doi: 10.1016/j.earlhumdev.2014.08.009.

2. Stoll BJ, Hansen NI, Bell EF, Walsh MC, Carlo WA, Shankaran S, et al. Trends in care practices, morbidity, and mortality of extremely preterm neonates, 19932012. JAMA. 2015;314(10):1039-1051; doi: 10.1001/ jama.2015.10244.

3. Kathegesu E, Beucher J, Daniel V, Guillot S, Lefeuvre S, Deneuville E, et al. Respiratory outcome of bronchopulmonary dysplasia in school-age children [in French]. Arch Pediatr. 2016;23(4):325-332; doi: 10.1016/j.arcped. 2015.12.013.

4. Tsopanoglou SP, Davidson J, Goulart AL, de Moraes Barros MC, dos Santos AMN. Functional capacity during exercise in very-low-birth-weight premature children. 
Pediatr Pulmonol. 2014;49(1):91-98; doi: 10.1002/ ppul.22754.

5. Powers SK, Howley ET. Exercise physiology: theory and application to fitness and performance, $8^{\text {th }}$ ed. New York: McGraw-Hill; 2015.

6. Agostini V, Nascimbeni A, Gaffuri A, Imazio P, Benedetti MG, Knaflitz M. Normative EMG activation patterns of school-age children during gait. Gait Posture. 2010;32(3):285-289; doi: 10.1016/j.gaitpost.2010.06.024.

7. Granata KP, Padua DA, Abel MF. Repeatability of surface EMG during gait in children. Gait Posture. 2005; 22(4):346-350; doi: 10.1016/j.gaitpost.2004.11.014.

8. National High Blood Pressure Education Program Working Group on High Blood Pressure in Children and Adolescents. The fourth report on the diagnosis, evaluation, and treatment of high blood pressure in children and adolescents. Pediatrics. 2004;114(Suppl. 2):555-576.

9. Von Leupoldt A, Mertz C, Kegat S, Burmester S, Dahme B. The impact of emotions on the sensory and affective dimension of perceived dyspnea. Psychophysiology. 2006;43(4):382-386; doi: 10.1111/j.1469-8986. 2006.00415.x.

10. Karila C, de Blic J, Waernessyckle S, Benoist MR, Scheinmann P. Cardiopulmonary exercise testing in children: an individualized protocol for workload increase. Chest. 2001;120(1):81-87; doi: 10.1378/chest. 120.1.81.

11. Wasserman K, Hansen JE, Sue DY, Stringer WW, Sietsema KE, Sun X-G. Principles of exercise testing and interpretation: including pathophysiology and clinical applications, $3^{\text {rd }}$ ed. Philadelphia: Lippincott Williams \& Wilkins; 2005.

12. Hermens HJFB, Merletti R, Rau G, Disselhorst-Klug C, Stegeman DF. HGS project. Recommendations for sensor locations on individual muscles; 2012. Available from: http://seniam.org/sensor_location.htm.

13. Downs JA, Edwards AD, McCormick DC, Roth SC, Stewart AL. Effect of intervention on development of hip posture in very preterm babies. Arch Dis Child. 1991; 66(7 Spec No):797-801; doi: 10.1136/adc.66.7_spec_ no.797.

14. Monterosso L, Kristjanson L, Cole J. Neuromotor development and the physiologic effects of positioning in very low birth weight infants. J Obstet Gynecol Neonatal Nurs. 2002;31(2):138-146; doi: 10.1111/j.15526909.2002.tb00033.x.

15. Merlo E, Pozzo M, Antonutto G, di Prampero PE, Merletti R, Farina D. Time-frequency analysis and estimation of muscle fiber conduction velocity from surface EMG signals during explosive dynamic contractions. J Neurosci Methods. 2005;142(2):267-274; doi: 10.1016/ j.jneumeth.2004.09.002.

16. Jacobs R, Bobbert MF, van Ingen Schenau GJ. Mechanical output from individual muscles during explosive leg extensions: the role of biarticular muscles. J Bio- mech. 1996;29(4):513-523; doi: 10.1016/0021-9290 (95)00067-4.

17. Skyrme AD, Cahill DJ, Marsh HP, Ellis H. Psoas major and its controversial rotational action. Clin Anat. 1999;12(4):264-265; doi: 10.1002/(SICI)1098-2353 (1999)12:4<264::AID-CA4>3.0.CO;2-L.

18. De Ruiter CJ, Elzinga MJH, Verdijk PWL, van Mechelen W, de Haan A. Changes in force, surface and motor unit EMG during post-exercise development of low frequency fatigue in vastus lateralis muscle. Eur J Appl Physiol. 2005;94(5-6):659-669; doi: 10.1007/s00421005-1356-x.

19. Balog EM, Thompson LV, Fitts RH. Role of sarcolemma action potentials and excitability in muscle fatigue. $\mathrm{J}$ Appl Physiol. 1994;76(5):2157-2162; doi: 10.1152/jappl. 1994.76.5.2157.

20. Pedersen TH, L-H Huang C, Fraser JA. An analysis of the relationships between subthreshold electrical properties and excitability in skeletal muscle. J Gen Physiol. 2011;138(1):73-93; doi: 10.1085/jgp.201010510.

21. Collins LM. Analysis of longitudinal data: the integration of theoretical model, temporal design, and statistical model. Annu Rev Psychol. 2006;57:505-528; doi: 10.1146/annurev.psych.57.102904.190146.

22. Bauman A, Ainsworth BE, Bull F, Craig CL, Hagströmer M, Sallis JF, et al. Progress and pitfalls in the use of the International Physical Activity Questionnaire (IPAQ) for adult physical activity surveillance. J Phys Act Health. 2009;6(Suppl. 1):S5-S8; doi: 10.1123/jpah. 6.s1.s5. 